\title{
Comparation of the eutectic mixture of lidocaine/prilocain versus benzocaine gel in children
}

\author{
Ana Maria Leyda ${ }^{1}$, Carmen Llena ${ }^{2^{*}}$ \\ ${ }^{1}$ Private practice in Pediatric Dentistry, Valencia, Spain; \\ ${ }^{2}$ Department of Stomatology, University of Valencia, Valencia, Spain. \\ Email: $\underline{\text { llena@uv.es }}$
}

Received 2 June 2011; revised 15 August 2011; accepted 25 August 2011.

\begin{abstract}
Objective: To compare the anesthetic effect of a non commercial eutectic mixture of $4 \%$ lidocaine/ prilocaine (PLO 4\%) and $20 \%$ benzocaine gel (Hurricaine $\left.{ }^{\circledR}\right)$, as topical anesthetic, prior to inferior alveolar nerve block and buccal infiltration anesthesia in 5 - 12 year old children. Study design: Infiltrative anesthesia was applied in $\mathbf{5 0}$ children, divided in two groups $(n=25)$ using PLO $4 \%$ and Hurricaine ${ }^{\circledR}$ as topical anesthesia prior to infiltration. Physical reactions were registered using the Sound-EyesMotor Scale. Physiological changes expressed by arterial pressure and heart rate. Subjective pain response was scored on a Facial Image Scale. Physical physiological and subjective response was related to the type of topical anesthetic, age and sex using $\chi^{2}$ and Mann-Whitney $U$ test. Results: Physical responses to puncture were similar and localized in the state of comfort with both anesthetics. Girls showed more ocular response than boys. Subjective pain perception and physiological reactions showed no anesthetic- or sex-related differences, except for heart rate before and after the procedure which was significantly higher in girls. Conclusions: PLO 4\% showed the same capacity as Hurricaine ${ }^{\circledR}$ in reducing pain response to needle puncture. Girls expressed more needle puncture-related pain than boys. The young children showed most prior comfort and less discomfort to the puncture than older children.
\end{abstract}

Keywords: Lidocaine; Prilocaine; Benzocaine; Topical Anesthetics

\section{INTRODUCTION}

In-office dental procedures are associated with discomfort and pain for most people. For the part of the population which has to visit the dentist, this association generates fear and anxiety. If the patient is a child, controlling any discomfort and/or pain in dental procedures is fundamental and key to ensuring pleasant, safe and effective treatment. For this purpose local anesthetics (LA) are routinely used in pediatric dentistry [1].

However, the application of local anesthesia is often frightening because it is associated with the use of needles, punctures and pain [2]. Together with cavity preparation by turbine, the application of anesthesia is one of the procedures which generate the greatest fear and anxiety in pediatric patients and dentists themselves [3]. Local anesthesia is, however, a basic technique in handling patient behavior because an effective anesthetic technique will provide a relaxed patient, quality, effective work and satisfied parents.

To mitigate the sensation of discomfort produced by needle insertion, various resources and procedures are used [4-8]; some are psychological procedures, such as distraction or suggestion, others physical procedures, such as warming the anesthetic solution, administering it very slowly $(1 \mathrm{ml} / \mathrm{min})$ to avoid the discomfort caused as the anesthetic liquid distends the tissue and injecting the anesthetics at a lower pressure of $306 \mathrm{~mm} / \mathrm{Hg}$ [5]. Mechanical resources are also used such as generating other simultaneous sensations to distract the child's attention and temporarily block the transmission of nociceptive messages ( $\mathrm{A} \delta$ and $\mathrm{C}$ fibres) or using needles with calibers below $\mathrm{N} 27$ because there is less perceived pain in the mandibular block technique with smaller caliber needles [6,7]. Another group of resources is based on the prior surface anesthetic of oral mucosa by cooling, transcutaneous electronic nerve stimulation or the use of topical anesthetics (TA) [8].

The high concentrations of TA diffuse through the epithelium and act on the mucosa nerve endings, blocking them. This decreases the painful sensation generated by needle insertion. Currently, TA can be found in different presentations and compositions. However, they still need to be combined with the other resources men- 
tioned above and even so, do not totally mitigate the discomfort generated by needle insertion when applying the local anesthetic [9].

Studies on topical anesthetics seek an agent which meets a series of characteristics indicated as "ideal". A powerful, low dose TA is needed which is rapidly absorbed by the keratinized and non-keratinized mucosa, remains in contact with the tissue to be anesthetized for a long time to increase the depth of the action of the agent and with characteristics which make it useful for pediatric use: pleasant flavor, smell, color and texture [8].

These studies focus on the design and evaluation of new TA preparations which meet these conditions [10] and the present study has a similar aim, as its purpose is to compare the anesthetic effect of a non commercial eutectic mixture of $4 \%$ lidocaine and prilocaine (LPO $4 \%$ as topical anesthetic on the oral mucosa, prior to inferior mandibular and buccal nerve block anesthesia in 5-12 year old children, comparing the results with $20 \%$ benzocaine gel (Hurricaine ${ }^{\circledR}$ ).

\section{MATERIAL AND METHODS}

\subsection{Subjects}

The study population was children between the ages of 5 and 12 who attended two private dental clinics in the province of Valencia, Spain and the Dental Clinic at the Faculty of Dentistry at CEU Cardenal Herrera University in the town of Moncada (Valencia, Spain) between May and November 2008.

The sample was chosen using a consecutive sampling system. Children who met the following criteria were included in the study: at least 5 years old but under the age of 13, needing clinical procedures which involved mandibular nerve block and understanding Spanish. Exclusion criteria were: having physical limitations which might alter or require modifications in the local troncular anesthesia, having psychological or sensorial limitations, emotional limitations or a history of non collaborative behavior with the dental treatment, having a systemic pathology which contraindicated the use of local anesthetics and arriving for treatment with pain or dental emergency.

\subsection{The Anesthetic and the Needle}

The anesthetics used in the study were $20 \%$ benzocaine gel (Hurricaine ${ }^{\circledR}$ ) and $4 \%$ lidocaine and prilocaine ointment (LPO 4\%), both prepared for this study in single dose 0.5 gram tubes.

Local anesthesia was applied in all cases with disposable sterile $25 \mathrm{~mm} 30$ caliber dental anesthesia needles (Normon jet plus ${ }^{\circledR}$ ).

\subsection{Measurement of Arterial Pressure, Heart Rate, Physical Reactions and Subjective Pain Perception}

Patient physiology was measured using a hand-held pulse oximeter (LTD810, Moretti) and a wrist device to measure arterial pressure (Omrom, RX 3).

Physical reactions were measured on the Sound-EyesMotor scale (Table 1) designed by Doctor Wright [11], a scale frequently used in pediatric dentistry studies as it enables an assessment of the relationship between pain and the reactions the sensation of pain generates in the patient's eyes, movements and verbal expressions of discomfort and also the degree of intensity of the sensation of pain [11].

Subjective pain perception was scored on the facial images scale (FIS) [12] (Figure 1) which has 5 faces with a number assigned from 1 to 5.1 coincides with the face expressing the most happiness and 5 with the most sadness. The intermediate numbers express intermediate emotions between these two. Scoring is towards which face the child most identifies with at that moment [12].

\subsection{Registered Variables}

The variables registered on the patient record were: sex, age, clinical center, variables used to evaluate patient physical reactions to puncture on the Sound-Eyes-Motor scale, variables to record each patient's subjective perception of puncture on the Facial Images Scale, variables related to physiological changes; heart rate, recorded at the beginning of the session, at the moment of puncture and arterial pressure recorded at the start and end of the session.

\subsection{Procedures}

In all the patients the process of adapting to the dental clinic was respected and treatments were progressively introduced by order of difficulty, but in all cases the appointment included in the study was the first mandibular nerve block done on the patient.

The type of topical anesthesia applied to each patient was chosen alternatively by the operator, without bearing in mind age and sex of the patients. All odd-numbered patients in the study received $20 \%$ benzocaine gel and all even-numbered patients received the LPO $4 \%$. All the anesthesia was carried out by the same operator unifying the manner of applying the injection and the pre- and post-injection instructions and information given to the patients. A prior study was carried out in 10 patients with the same characteristics to systematize and protocolize the process.

We take in mind ethical considerations of Helsinki declaration in 1975 revised in 2000. Before the start of the session and after checking that the child met the cri- 
Table 1. Sound-Eyes-Motor scale [12].

\begin{tabular}{|c|c|c|c|c|}
\hline Observations & 1.- Comfort & 2.-Slight discomfort & 3.- Moderate pain & 4.-Pain \\
\hline Sound & $\begin{array}{l}\text { No sounds } \\
\text { indicating pain }\end{array}$ & $\begin{array}{l}\text { Non specific sounds which may } \\
\text { indicate pain. }\end{array}$ & $\begin{array}{l}\text { Specific verbal complaints } \\
\text { raising voice. }\end{array}$ & $\begin{array}{l}\text { Verbal complaints } \\
\text { indicating intense pain. }\end{array}$ \\
\hline Eyes & $\begin{array}{l}\text { No ocular signs of } \\
\text { pain }\end{array}$ & $\begin{array}{l}\text { - } \quad \text { Eyes wide open. } \\
\text { - } \quad \text { Shows concern. } \\
\text { - No tears. }\end{array}$ & $\begin{array}{l}-\quad \text { Watery eyes. } \\
\text { - } \quad \text { Blinking eyes. }\end{array}$ & $\begin{array}{l}-\quad \text { Crying. } \\
-\quad \text { Tears rolling } \\
\text { down the face. }\end{array}$ \\
\hline Motor & $\begin{array}{l}\text { - } \quad \text { Relaxed hands } \\
\text { - } \quad \text { Body } \\
\text { apparently relaxed. }\end{array}$ & $\begin{array}{ll}\text { - } & \text { Hands show stress or tension. } \\
\text { - } & \text { Grasps hold of chair. } \\
\text { - } & \text { Muscular tension. }\end{array}$ & $\begin{array}{l}\text { - } \quad \text { Arm or body movements } \\
\text { with no aggressive intention } \\
\text { - } \quad \text { Physical contact. } \\
\text { - } \quad \text { Pulling faces or grimacing. }\end{array}$ & $\begin{array}{l}\text { Hand movement for } \\
\text { aggressive contact. }\end{array}$ \\
\hline
\end{tabular}
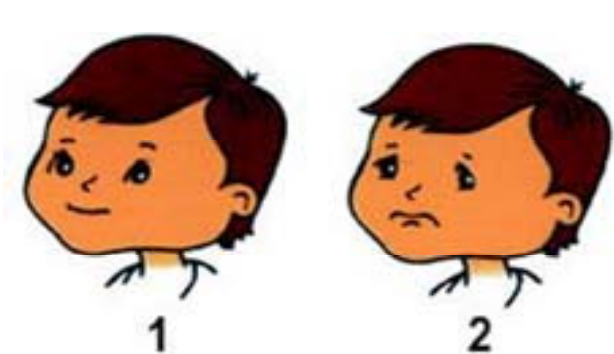

Figure 1. Facial image scale [13].

teria for inclusion in the study, the father, mother or guardian was given an information sheet and a verbal explanation by the operator. After the information, informed consent was requested and when signed was added to the patient data record card.

When the patient was comfortably seated in the dental chair, the operator asked about his/her level of pain and feeling of comfort at that moment, the assistant took the arterial pressure and checked heart rate with the pulse oximeter. Then the patient was given the necessary instructions and information about all procedures and placed in the supine position in the dental chair. The operator dried the area for topical anesthetic with sterile gauze for 60 seconds to eliminate the saliva and mucins which cover oral mucosa and the keratinzed layer of the epithelium was swabbed to favor subsequent TA absorption. While the operator dried the mucosa, the assistant collected the content of the single dose of TA on a swab. The operator applied the TA on the dry and swabbed area for 2 minutes, placing an aspirator in lingual side of the jaw to prevent TA contact with saliva and movement in the place of application. After the established time, the area was washed with abundant water to eliminate TA remains.

Local anesthesia was administered using the distraction technique, preventing the patient from seeing the needle. The puncture was made with a vibrating movement of the cheek; at that moment the assistant recorded the heart rate marked on the pulse oximeter. This value, together with ocular, motor or verbal reaction generated at the moment of the puncture, was recorded on the card. On finishing injection of local anesthetic, the pulse oximeter was withdrawn from the patient's finger and the patient was allowed to rinse his/her mouth. When the treatment had finished, the operator asked the patient again about his/her level of pain and feeling of comfort at that moment, and the assistant took his/her arterial pressure. All the values were recorded on the card.

\subsection{Statistical Analysis}

Data were analyzed using SPSS 15.0 software (SPSS, Inc, Chicago, Ill); a descriptive analysis of the results was made. Age was recoded in two groups: children from 5 to 8 years old, and children from 9 to 12 years old. The variables for physical reactions and the patient's subjective sensation and the type of anesthesia were compared with recoded age, sex and type of anesthetic using the Chi-Square test. Mann-Whitney's U test was used to relate physiological reactions with sex, recoded age and type of anesthesia, for a confidence level of $95 \%$.

\section{RESULTS}

\subsection{Sample Description}

Of the 53 patients chosen to take part in the study, 3 were not included because of a background of disruptive behavior in office, the three were girls and so a total of 50 individuals took part in the study: 24 males (48\%) and 26 females (52\%) between the ages of 5 years and one month and 12 years and 11 months, with an average 
age of 8 years and 5 months (standard deviation $=2.29$ ). During treatment, half the patients received benzocaine gel as TA and the other half, LPO 4\%.

Physical reactions to puncture were grouped according to the three variables in the sound-eyes-motor scale. For the variable sound, $90 \%$ (45) of the participants had expressions compatible with well being; of the remaining 10\%, 4 had expressions compatible with slight discomfort and one with moderate pain. For the variable "eyes", 90\% (45) of the patients had reactions compatible with well being; of the remaining 10\%, 4 showed reactions compatible with slight discomfort and one with moderate pain. Finally, for the variable "motor", 86\% (43) of the patients had reactions compatible with well being; of the remaining 14\%, 4 showed reactions compatible with slight discomfort and 3 with moderate pain.

The patients' own perception of well being and pain, collected on the facial image scale gave the following results: $86 \%$ of the patients (43) felt very well when they sat down on the dental chair and had no pain (value 1 on the scale). The remaining $14 \%$ (7) felt simply well (value 2 on the scale). At the end of the session $74 \%$ of the patients (37) felt very well and without pain (value 1 on the scale); while 22\% (11) felt simply well (value 2 on the scale) and $4 \%$ felt not well (value 3 on the scale).

\subsection{Relationship between Anesthetic and Physical Reactions}

The variables registered on the sound-eyes-motor scale showed no significant differences in relation to the anesthetic used with 90\% in the situation of comfort (Table 2).

\subsection{Relationship between Sex and Physical Reactions}

Analysis by sex, shows a greater motor, ocular and ver- bal reaction to puncture in females than males, and acquired statistical significance in the variable "eyes" where it was found that males experience significantly greater comfort than females $(p=0.03)$ (Table 2).

\subsection{Relationship between Age and Physical Reactions}

Using the "sound, eyes, motor" scale for the expression of discomfort through sounds, no 5 - 8 years old children showed discomfort after puncture, but 21.1\% (4) of the 9 - 12 years old children expressed a uncomfortable perception through unspecific sounds ( $\mathrm{p}=0.017)$.

\subsection{Relationship between Anesthetic and Subjective Perception of Pain}

Comparison of the two TA, in relation to the subjective perception of pain and comfort recorded by applying the facial image scale at the beginning and end of treatment gave practically the same results, mainly located in the state of comfort (Table 3).

\subsection{Relationship between Sex and Subjective Perception of Pain}

The results were also very similar for males and females mainly located in the state of comfort (Table 3).

\subsection{Relationship between Age and Subjective Perception of Pain}

Depending on the age of patients, we found a significant association for comfort perception and pain, through the pain facial scale used; so, $96.98 \%$ of the 5 - 8 years old children (30) felt very well and without pain when sited at the clinical chair (score 1 from the scale); the rest, $3.2 \%$ (1), felt simply well (score 2); while in the group of 9 - 12 years old, $68.4 \%$ of the children showed a score 1 and $31.6 \%$ a score $2(\mathrm{p}=0.009)$.

Table 2. Comparison by type of anesthestic and sex of the Sound-Eyes-Motor scale's scoring (significant value in bold).

\begin{tabular}{|c|c|c|c|c|c|}
\hline & $\mathrm{n}$ & Comfort & Slight discomfort & Moderate pain & $\mathrm{p}$ \\
\hline & & & SOUND & & \\
\hline LPO4\% & 25 & $23(92 \%)$ & $1(4 \%)$ & $1(4 \%)$ & 0.52 \\
\hline Hurricaine & 25 & 22 (88\%) & $3(12 \%)$ & 0 & \\
\hline Male & 24 & $23(95.8 \%)$ & $1(4.2 \%)$ & 0 & 0.34 \\
\hline \multirow[t]{2}{*}{ Female } & 26 & $22(84.6 \%)$ & $3(11.5)$ & $1(3.8 \%)$ & \\
\hline & & & EYES & & \\
\hline LPO4\% & 25 & $23(92 \%)$ & $2(8 \%)$ & 0 & 0.62 \\
\hline Hurricaine & 25 & $22(88 \%)$ & $2(8 \%)$ & $1(4 \%)$ & \\
\hline Male & 24 & 24 (100\%) & 0 & 0 & 0.02 \\
\hline \multirow[t]{2}{*}{ Female } & 26 & 21 (80.8\%) & $4(15.4 \%)$ & $1(3.8 \%)$ & \\
\hline & & & MOTOR & & \\
\hline LPO4\% & 25 & $22(88 \%)$ & $1(4 \%)$ & $2(8 \%)$ & 0.51 \\
\hline Hurricaine & 25 & $21(84 \%)$ & $3(12 \%)$ & $1(4 \%)$ & \\
\hline Male & 24 & $23(95.8 \%)$ & $1(4.2 \%)$ & 0 & 0.14 \\
\hline Female & 26 & $20(76.9 \%)$ & $3(11.5 \%)$ & $3(11.5 \%)$ & \\
\hline
\end{tabular}


Table 3. Subjective perception of pain using the "Facial Image Scale" by type of anesthetic and sex.

\begin{tabular}{|c|c|c|c|c|c|c|c|}
\hline & $\mathbf{n}$ & 1 & 2 & 3 & 4 & 5 & $\mathbf{p}$ \\
\hline & \multicolumn{7}{|c|}{ INITIAL PERCEPTION } \\
\hline LPO4\% & 25 & $21(84 \%)$ & $4(16 \%)$ & 0 & 0 & 0 & \multirow{2}{*}{0.50} \\
\hline Hurricaine & 25 & $23(88 \%)$ & $3(12 \%)$ & 0 & 0 & 0 & \\
\hline Male & 24 & 22 (91.7\%) & $2(8.3 \%)$ & 0 & 0 & 0 & \multirow{2}{*}{0.24} \\
\hline \multirow[t]{2}{*}{ Female } & 26 & $21(80.8 \%)$ & $5(19.25 \%)$ & 0 & 0 & 0 & \\
\hline & & & FINAL PER & & & & \\
\hline LPO4\% & 25 & $20(83 \%)$ & $4(16 \%)$ & 0 & 0 & 0 & \multirow{2}{*}{0.36} \\
\hline Hurricaine & 25 & 17 (68\%) & $7(28 \%)$ & $1(4 \%)$ & 0 & 0 & \\
\hline Male & 24 & $18(75 \%)$ & $5(20.8 \%)$ & $1(4.2 \%)$ & 0 & 0 & \multirow{2}{*}{0.57} \\
\hline Female & 26 & 19 (76\%) & $6(24 \%)$ & 0 & 0 & 0 & \\
\hline
\end{tabular}

\subsection{Relationship between Anesthetic and Arterial Pressure Modification}

The initial and final measurement of arterial pressure provided six values for each patient: systolic pressures (initial and final) and the difference between the two; two diastolic pressures (initial and final) and the difference between the two. After the procedure a clear modification of arterial pressure was observed but with no significant differences between them (Table 4).

\subsection{Relationship between Sex and Arterial Pressure Modification}

In relation to sex, patients of both sexes experienced similar modifications in arterial pressure (Table 4).

\subsection{Relationship between Anesthetic and Heart Rate Modification}

Analysis of heart rate modification on puncture gave similar results. After the procedure a clear modification of heart rate was observed but with no significant differences between them (Table 5).

\subsection{Relationship between Sex and Heart Rate Modification}

Difference between initial and final heart rate was significantly higher in females $(\mathrm{p}=0.04)$ (Table 5).

\section{DISCUSSION}

Although currently the effectiveness of TA in reducing pain associated to intraoral injection of LA is much debated, it is certainly much used in pediatric dentistry [13].

Multiple factors are involved in pain perception, including psychological factors such as personality, fear, anxiety and the sensation of control over pain producing agents or pain itself [2].

The results of this present study showed no difference between the puncture pain felt and perceived with the two TA studied.
$20 \%$ Benzocaine gel is an ester-type anesthetic, with a fast onset of action of approximately 30 seconds [13,14], a pleasant taste, a longer duration than other TA and a low level of systemic absorption making it safe for use in children [11]. However, its main disadvantage is low bioadhesivity $[4,15,16]$.

$\mathrm{LPO} 4 \%$ is a non commercial eutectic mixture of $4 \%$ lidocaine and prilocaine. Both these anesthetics, which belong to the amide family, are less likely to provoke allergic reactions than ester anesthetics and are extremely similar to the anesthetic used for infiltration anesthesia (2\% lidocaine with epinephrine at 1:100,000), also an amide. The galenic formula of LPO4\% is achieved with high occlusive, self-emulsifying bases, thanks to the inclusion of ethoxylated lanolin and a commercialized cosmetic base oil in water emulsion. It is a greasy product which waterproofs the mucosa, favoring greater localization and concentration of the product in the area to be anesthetized. The euctectic mixture of lidocaine and prilocaine significantly increases the anesthetic power of the two components individually and solves the problem of solubility in these anesthetic molecules so they can be included in their most effective but least soluble base form, the only form which can cross the nervous fiber membrane, and so pKa must be greater than 7.4. The topical anesthetic "LPO4\%" has a $\mathrm{pH}$ of 8.4 [13]. Its pink color and strawberrypineapple taste make it acceptable to the pediatric population [16]. As LPO4\% is a non commercialized anesthetic preparation there are no previous studies on its effectiveness in comparison to other TA for the same uses. In contrast, there are many studies on benzocaine gel as it is currently the most popular topical anesthetic, the most used and the most recommended in general and pediatric dentistry $[13,16]$ as it is well accepted by children and professionals.

Studies in children show in some cases a slight superiority of $20 \%$ benzocaine gel over other agents $[13,17]$. 
In contrast, some studies show greater effectiveness against pain of other agents, in particular 20\% lidocaine patches [16]. Other studies found that $20 \%$ benzocaine gel was effective but not more than the other agents which it was compared with $[9,14]$. This present study can be included in this group of studies as it compares the effectiveness of reducing injection pain of $20 \%$ benzocaine gel and the eutectic mixture of $4 \%$ lidocaine and prilocaine in a group of children between the ages of 5 and 12. The authors found no objective evidence for the greater effectiveness of either of the two agents in reducing needle insertion pain.

Table 4. Arterial pressure changes by type of anesthetic and sex. ISP: initaial systolic presure, FSP: final systolic presure, IDO: initial diastolic presure, FDP: final diastolic presure.

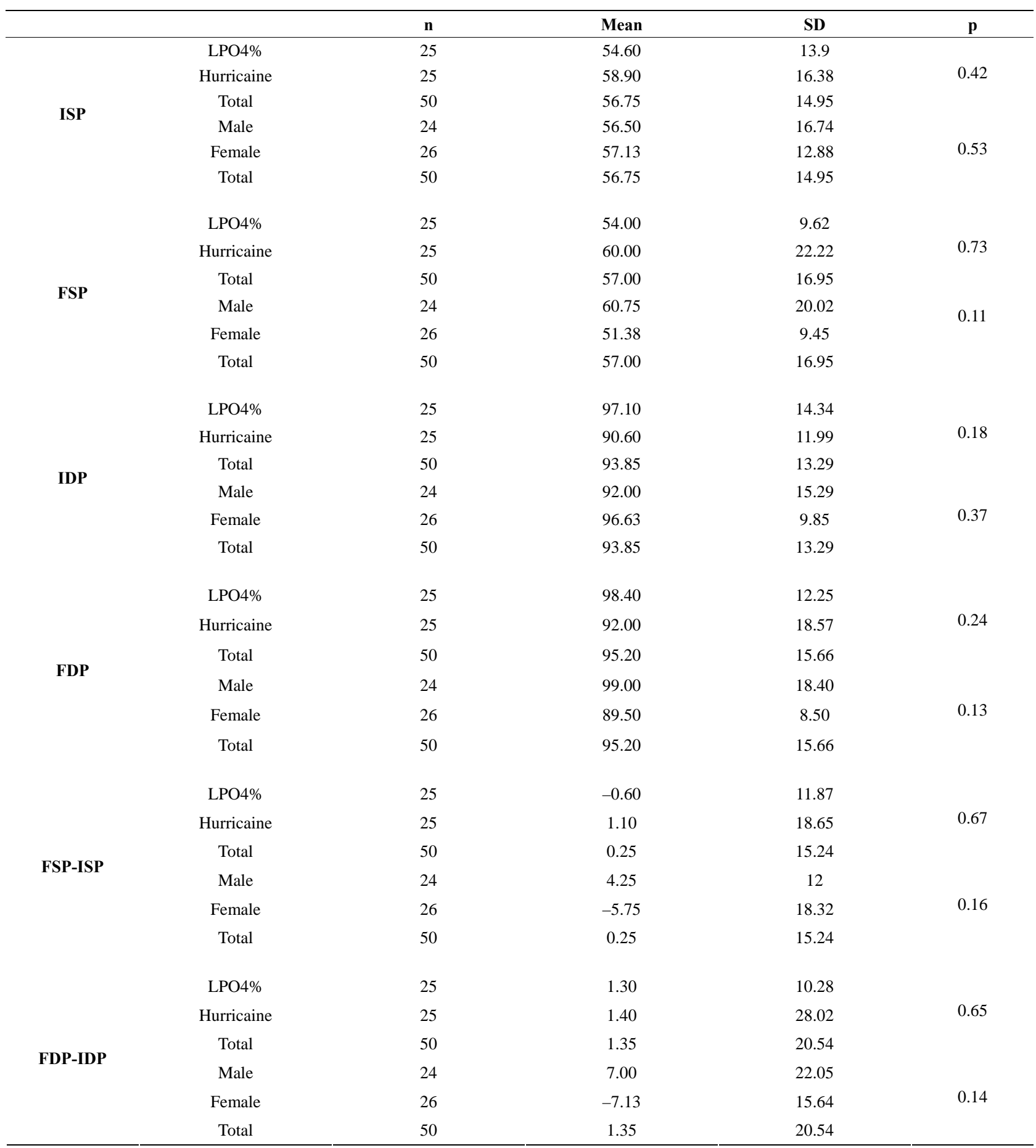


Table 5. Heart rate modification by type of anesthetic and sex IHR: initial hearth rate, FHR: final heart rate.

\begin{tabular}{|c|c|c|c|c|c|}
\hline & & $\mathbf{n}$ & Mean & SD & $\mathbf{p}$ \\
\hline \multirow{6}{*}{ IHR } & LPO4\% & 25 & 80.90 & 9.32 & \multirow[b]{2}{*}{0.18} \\
\hline & Hurricaine & 25 & 83.70 & 14.97 & \\
\hline & Total & 50 & 82.30 & 12.22 & \multirow{4}{*}{0.64} \\
\hline & Male & 24 & 82.75 & 10.00 & \\
\hline & Female & 26 & 81.63 & 15.73 & \\
\hline & Total & 50 & 82.30 & 12.22 & \\
\hline \multirow{6}{*}{ FHR } & LPO4\% & 25 & 91.70 & 18.99 & \multirow[b]{2}{*}{0.27} \\
\hline & Hurricaine & 25 & 92.80 & 12.77 & \\
\hline & Total & 50 & 92.25 & 15.76 & \multirow{4}{*}{0.23} \\
\hline & Male & 24 & 88.92 & 11.09 & \\
\hline & Female & 26 & 97.25 & 20.82 & \\
\hline & Total & 50 & 92.25 & 15.76 & \\
\hline \multirow{6}{*}{ FHR-IHR } & LPO4\% & 25 & 10.80 & 17.56 & \multirow{3}{*}{0.85} \\
\hline & Hurricaine & 25 & 9.10 & 13.49 & \\
\hline & Total & 50 & 9.95 & 15.27 & \\
\hline & Male & 24 & 6.17 & 10.84 & \multirow{3}{*}{0.23} \\
\hline & Female & 26 & 15.63 & 19.68 & \\
\hline & Total & 50 & 9.95 & 15.27 & \\
\hline
\end{tabular}

Although our study shows no objective evidence of greater effectiveness of LPO4\% than Hurricaine ${ }^{\circledR}$ in reducing needle insertion pain; it was not found to be less efficient and, therefore, more studies are required to analyze other parameters such as greater safety, as it is fully similar to the anesthesia used for infiltration anesthesia and has a greater capacity to waterproof the mucosa favoring better localization and concentration of product in the area to be anesthetized, preventing it from being mixed with saliva. This would solve the greatest problem found with $20 \%$ benzocaine gel which is the lack of bioadhesivity to the oral mucosa which generates movement from the site of application to the surrounding mucosa, reducing the anesthetic effect on the tissue and sometimes causing patient discomfort $[4,15,16]$. Another possible advantage of LPO4\% is its pink color and strawberry-pineapple taste which would favor acceptance by the pediatric population.

Analysis of the data has shown an interesting relationship between sex of the participants and their reaction to puncture of the mucosa previously treated with TA, with more intense ocular reaction in females than males. In our study, 5 of the 26 girls showed ocular and motor expressions which did not coincide with comfort, and 4 of them were between the ages of 7 and 9 . No boy at that age showed any level of discomfort during the local anesthesia procedure.

The children between 5 and 12 years old show typical characteristics of this stage in the different areas of development, such as desire for productive work, thereby increasing feelings of competition; children are afraid of the imaginary, of bodily harm, loss of image and deteriorated self-esteem. They are intensely preoccupied and anxious about imaginary rather than real causes.

Related to dental fear, differences between sexes seem to be inexistent, at least in the occidental societies, although studies show different results, sex and age are in fact co-factors modulated by other variables such as the patient's culture, socio-economic situation, etc. In fact, only after reaching certain ages do differences in behavior and anxiety levels among girls and boys become evident [18,19]. Taylor et al. compared the behavior of boys and girls and only after the age of 7 and during the application of local anesthesia did they find that boys showed fewer expressions of discomfort and negative behaviors than girls [20].

Studies concluded that girls showed more fear than boys. Not that they felt more fear, but that they showed it, because boys behave better than girls when they are given strict instructions about how to behave [21], 
showing that girls had significantly higher levels of dental anxiety than boys even in adolescent patients with greater levels of personal maturity [22].

\section{CONCLUSIONS}

As a result of this study we can conclude that LPO4\% was as capable as Hurricaine ${ }^{\circledR}$ in reducing pain generated by needle puncture for local anesthesia infiltration. When faced with needle puncture for local infiltration anesthesia by mandibular and buccal nerve block in 5 to 12 years old, girls expressed more pain by ocular expression than boys and a significant increase in heart rate was found in girls at the end of the therapeutic treatment. By age, children from 9 to 12 years old expressed more discomfort by sound expression than children from 5 to 8 years old. In the same way the younger children expressed more wellness before the procedure than older children.

\section{REFERENCES}

[1] Meechan, J.G. (2009) Pain control in local analgesia. European Archives of Paediatric Dentistry, 10, 71-76.

[2] Kudo, M. (2005) Initial injection pressure for dental local anesthesia: Effects on pain and anxiety. Anesthesia Progress, 52, 95-101. doi:10.2344/0003-3006(2005)52[95:IIPFDL]2.0.CO;2

[3] Peretz, B. and Efrat, J. (2000) Dental anxiety among young adolescent patients in Israel. International Journal of Paediatric Dentistry, 10, 126-132. doi:10.1046/j.1365-263x.2000.00181.x

[4] Hersh, E., Houpt, M.I., Cooper, S.A., Fedman, R.S., Wolf, M.S. and Levin, L.M. (1996) Analgesic efficacy and safety of an intraoral lidocaine patch. Journal of the American Dental Association, 127, 1626-1634.

[5] Ram, D. and Peretz, B. (2001) Administering local anaesthesia to paediatric dental patients-current status and prospects for the future. International Journal of Paediatric Dentistry, 12, 80-89. doi:10.1046/j.1365-263X.2002.00343.x

[6] Ram, D., Hermida, B.L. and Amir, E. (2007) Reaction of children to dental injection with 27- or 30-gauge needles. International Journal of Paediatric Dentistry, 17, 383-387. doi:10.1111/j.1365-263X.2007.00860.x

[7] Flanagan, T., Wahl, M.J., Schmitt, M.M. and Wahl, J.A. (2007) Size doesn't matter: Needle gauge and injection pain. General Dentistry, 55, 216-217.

[8] Meechan, J.G. (2002) Effective topical anesthetic agent and techniques. Dental Clinics of North America, 46, 759766. doi:10.1016/S0011-8532(02)00035-6

[9] Kreider, K.A., Stratmann, R.G., Milano, M., Agostini, F. and Munsell, M. (2001) Reducing children's injection pain: Lidocaine patches versus topical benzocaine gel. Paediatric Dentistry, 23, 19-23.

[10] Kohli, K., Crout, R. and Linscott, C. (2000) A survey of local and topical anesthesia use by pediatric dentists in the United States. Paediatric Dentistry, 23, 265-269.

[11] Wright, G.Z., Weinberger, S.J., Marti, R. and Plotzke, O. (1991) The effectiveness of infiltration anesthesia in the mandibular primary molar region. Paediatric Dentistry, 113, 278-283.

[12] Buchanani, H. and Niven, N. (2002) Validation of a Facial Image Scale to asses child dental anxiety. International Journal of Paediatric Dentistry, 12, 47-52.

[13] Primosch, R.E. and Rolland-Asensi, G. (2001) Comparison of topical EMLA 5\% oral adhesive to benzocaine $20 \%$ on the pain experienced during palatal anesthetic infiltration in children. Paediatric Dentistry, 23, 11-14.

[14] Stecker, S.S., Swift, J.Q., Hodges, J.S. and Erickson, P.R. (2002) Should a mucoadhesive patch (Dentipatch ${ }^{\circledR}$ ) be for gingival anesthesia in children? Anesthesia Progress, 49, 3-8.

[15] Lim, S. and Julliard, K. (2001) Evaluating the efficacy of EMLA topical anesthetic in sealant placement with rubber dam. Paediatric Dentistry, 26, 497-500.

[16] Wu, S.J. and Julliard, K. (2003) Children's preference of benzocaine gel versus the lidocaine patch. Paediatric Dentistry, 25, 401-405.

[17] Tulga, F. and Mutlu, Z. (1999) Four types of topical anesthetic agents: Evaluation of clinical effectiveness. Journal of Clinical Paediatric Dentistry, 23, 217-220.

[18] Folayan, M.O., Idehen, E.E. and Ojo, O.O. (2004) The modulating effect of cultura on the expresión of dental anxiety in children: A literatura review. International Journal of Paediatric Dentistry, 19, 225-232.

[19] Corkey, B. and Freeman, R. (1994) Predictors of dental anxiety in six-year-old children: Findings from a pilot study. Journal of Dentistry for Children, 5, 267-271.

[20] Taylor, M.H., Moyer, I.N. and Peterson, D.S. (1983) Effect of appointment time, age and gender on children's behavior in dental setting. Journal of Dentistry for Children, 50, 106-110.

[21] Malamed, S. (2009) What's new in local anaesthesia? Society for the Advancement of Anaesthesia in Dentistry, 25, 4-14.

[22] Kawamura, M., Takase, N., Sasahara, H. and Okada, M. (2008) Teenagers' oral health attitudes and behavior in Japan: Comparison by sex and age group. Journal of Oral Science, 2, 167-174. doi:10.2334/josnusd.50.167 